\title{
A hybrid optoelectronic Mott insulator ${ }^{\circledR}$
}

Cite as: Appl. Phys. Lett. 118, 141901 (2021); https://doi.org/10.1063/5.0044066

Submitted: 13 January 2021. Accepted: 19 February 2021. Published Online: 05 April 2021

H. Navarro, (D) J. del Valle, Y. Kalcheim, (D) N. M. Vargas, (D) C. Adda, (D) M.-H. Lee, P. Lapa, (D) A. Rivera-Calzada, (D) I. A. Zaluzhnyy, E. Qiu, O. Shpyrko, (D) M. Rozenberg, (D) A. Frano, and Ivan K. Schuller

\section{COLLECTIONS}

EP This paper was selected as an Editor's Pick
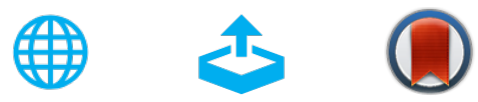

\section{ARTICLES YOU MAY BE INTERESTED IN}

Identification of spin-dependent thermoelectric effects in metamagnetic FeRh/heavy-metal bilayers

Applied Physics Letters 118, 142401 (2021); https://doi.org/10.1063/5.0038150

Spin-orbit torques: Materials, physics, and devices

Applied Physics Letters 118, 120502 (2021); https://doi.org/10.1063/5.0039147

Half-Heusler thermoelectric materials

Applied Physics Letters 118, 140503 (2021); https://doi.org/10.1063/5.0043552

\section{Challenge us.}

What are your needs for periodic signal detection?

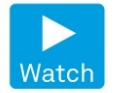

- Zurich

Instruments



( 


\title{
A hybrid optoelectronic Mott insulator 줄
}

\author{
Cite as: Appl. Phys. Lett. 118, 141901 (2021); doi: 10.1063/5.0044066 \\ Submitted: 13 January 2021 - Accepted: 19 February 2021 . \\ Published Online: 5 April 2021
}

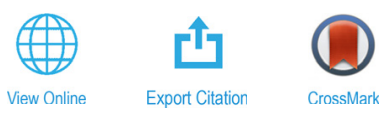

\author{
H. Navarro, ${ }^{1, a)}$ (D) J. del Valle, ${ }^{1,2}$ (D) Y. Kalcheim, ${ }^{1,3}$ N. M. Vargas, ${ }^{1}$ (D) C. Adda, ${ }^{1}$ (D) M.-H. Lee, ${ }^{1}$ (D) P. Lapa, \\ A. Rivera-Calzada, ${ }^{4}$ (D) I. A. Zaluzhnyy, (iD E. Qiu, ${ }^{7}$ O. Shpyrko, ${ }^{7}$ M. Rozenberg, ${ }^{5}$ (D) A. Frano, (D) and Ivan K. Schuller
}

\begin{abstract}
AFFILIATIONS
'Department of Physics, Center for Advanced Nanoscience, University of California, San Diego, California 92093, USA

${ }^{2}$ Department of Quantum Matter Physics, University of Geneva, 24 Quai Ernest-Ansermet, CH-1211 Geneva, Switzerland

${ }^{3}$ Faculty of Materials Science and Engineering, Technion-Israel Institute of Technology, 32000 Haifa, Israel

${ }^{4}$ Departamento de Física de Materiales, Universidad Complutense de Madrid, 28040 Madrid, Spain

${ }^{5}$ Laboratoire de Physique des Solides, CNRS, Université Paris Saclay, 91405 Orsay Cedex, France
\end{abstract}

a) Author to whom correspondence should be addressed: hnavarro@physics.ucsd.edu

\begin{abstract}
The coupling of electronic degrees of freedom in materials to create "hybridized functionalities" is a holy grail of modern condensed matter physics that may produce versatile mechanisms of control. Correlated electron systems often exhibit coupled degrees of freedom with a high degree of tunability which sometimes lead to hybridized functionalities based on external stimuli. However, the mechanisms of tunability and the sensitivity to external stimuli are determined by intrinsic material properties which are not always controllable. A Mott metalinsulator transition (MIT) is technologically attractive due to the large changes in resistance, tunable by doping, strain, electric fields, and orbital occupancy but not, in and of itself, controllable with light. Here, an alternate approach is presented to produce optical functionalities using a properly engineered photoconductor/strongly correlated hybrid heterostructure. This approach combines a photoconductor, which does not exhibit an MIT, with a strongly correlated oxide, which is not photoconducting. Due to the intimate proximity between the two materials, the heterostructure exhibits giant volatile and nonvolatile, photoinduced resistivity changes with substantial shifts in the MIT transition temperatures. This approach can be extended to other judicious combinations of strongly correlated materials.
\end{abstract}

Published under license by AIP Publishing. https://doi.org/10.1063/5.0044066

The physical properties of strongly correlated materials appeal to a broad scientific community because of the versatility and tunability of their electronic responses via internal and/or external perturbations. ${ }^{1-3}$ However, the number of ways of controlling a single correlated material is limited by the available internal degrees of freedom. This presents a difficult challenge when a device concept requires a specific mechanism of control that is not accessible within a material. Here, we present a simple, general solution to this limitation by judiciously designing a heterostructure that hybridizes the functionalities of two seemingly unrelated materials: a strongly correlated Mott insulator with a photoconducting semiconductor. This provides exquisite control of a metal-to-insulator transition (MIT) via optical means. With its striking simplicity, our methodology can be expanded to create hybridized functionalities across, e.g., semiconductors, strongly correlated, magnetic, and topological materials.

The Mott MIT in vanadium oxides-a hallmark of strong correlations-exemplifies behavior that is attractive for its fundamental interest and potential applications in modern technologies. ${ }^{2,4-10}$ This transition is characterized by an abrupt change in the electrical resistivity by several orders of magnitude and can be controlled by modulating the temperature, doping, pressure, electric field, and to a small extent with laser light. ${ }^{11-24}$ Some materials, such as $\mathrm{VO}_{2}$, also feature large changes in their optical properties across the MIT, making them attractive for optoelectronic applications. ${ }^{25-27}$ Earlier studies ${ }^{28,29}$ used $\mathrm{VO}_{2}$ to modify the boundary conditions in heterostructures and this way to implement electrically controlled optical devices. The reverse effect, i.e., controlling the Mott insulating transition by applying light, remains difficult because the effect of light is limited to small changes in the MIT. ${ }^{30}$

We have developed a highly sensitive optoelectronic Mott insulator, by heterostructuring vanadium oxide with a well-known semiconducting photoconductor. In this geometry (Fig. 1), light strongly affects the MIT through proximity effects, due to changes in the interfacial Schottky barrier, carrier doping, and/or light-induced interfacial catalytic reactions. We used the archetypical photoconductor, cadmium sulfide (CdS), one of the most studied chalcogenide semiconductor materials ${ }^{31}$ which has a high concentration of donor carriers. ${ }^{32}$ When exposed to visible light, CdS changes its conductivity 


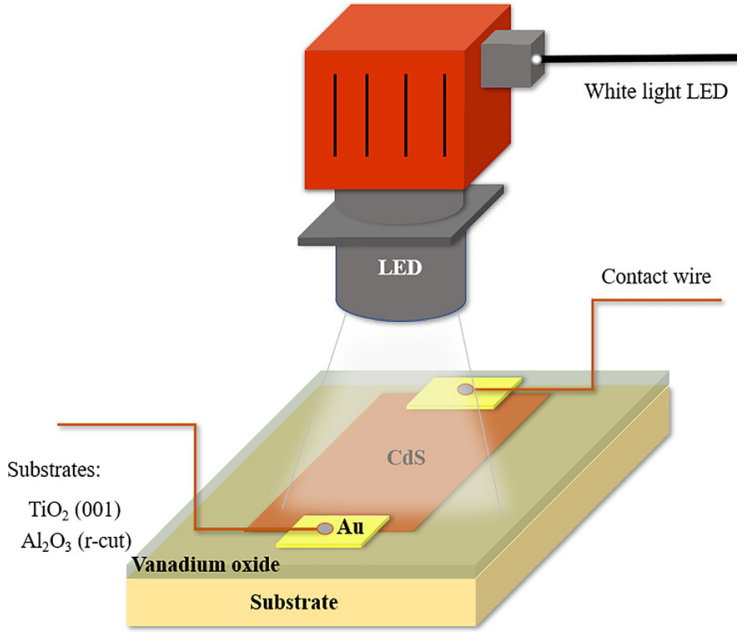

FIG. 1. Schematic device and experimental setup. A CdS $(80 \pm 1 \mathrm{~nm})$ film was deposited over vanadium oxide thin films $\left(10 \pm 1 \mathrm{~nm}\right.$ both). The $\mathrm{CdS} / \mathrm{N}_{2} \mathrm{O}_{3}$ and $\mathrm{CdS} / \mathrm{NO}_{2}$ heterostructures were illuminated with a white light LED. $\mathrm{CdS} / \mathrm{N}_{2} \mathrm{O}_{3}$ heterostructures were directly grown on $\mathrm{Al}_{2} \mathrm{O}_{3}$ (R-cut) substrates, and $\mathrm{CdS}_{2} \mathrm{O}_{2}$ were grown on a $\mathrm{TiO}_{2}$ (001) substrate for optimal growth. The bilayer conductivity was measured with two probes contacted by two Au pads.

substantially by generating electron and hole carriers ${ }^{33}$ with electrons as the main carriers in the photoexcited state. Moreover, when the $\mathrm{CdS}$ interacts with oxygen and light, this material can behave as a photocatalyst. $^{34,35}$ To maximize the effect of the interface on the transport properties, the thickness of the $\mathrm{VO}_{\mathrm{X}}$ was reduced to $10 \mathrm{~nm}$. We show that these hybrid heterostructures exhibit very large, volatile, and nonvolatile photoinduced modulations of both the amplitude and the critical temperature of the MIT. Moreover, there are interesting similarities and differences between the two VOx-based heterostructures. Such significant enhancement of the photosensitivity to light in Mott insulators provides an advanced material for basic research studies and a promising platform for optoelectronic applications.

We fabricated $10 \pm 1 \mathrm{~nm}$ thick epitaxial $\mathrm{V}_{2} \mathrm{O}_{3}$ film on top of $\mathrm{r}$-cut sapphire substrates using rf magnetron sputtering from a $\mathrm{V}_{2} \mathrm{O}_{3}$ target, in an 8 mTorr high-purity argon (>99.999\%) atmosphere. The substrate temperature during deposition is $720^{\circ} \mathrm{C}$, and the sample is cooled at a rate of $80^{\circ} \mathrm{C} / \mathrm{min}$ after growth. A $10 \pm 1 \mathrm{~nm}$ thick epitaxial $\mathrm{VO}_{2}$ film was grown by reactive sputtering on top of an $\mathrm{TiO}_{2}$ substrate (oriented along the (001) plane). A 4 mTorr argon/oxygen mix (8\% $\mathrm{O}_{2}$ ) was used during deposition, and the substrate was kept at $600^{\circ} \mathrm{C}$ during the growth and later cooled down at a rate of $12^{\circ} \mathrm{C} / \mathrm{min}$. CdS $80 \pm 1 \mathrm{~nm}$ thick film was grown on top of the VOx with rf magnetron sputtering from a CdS target, in a 2 mTorr pure argon atmosphere at $150^{\circ} \mathrm{C}$. Electrical transport properties of a CdS film grown directly on $\mathrm{Al}_{2} \mathrm{O}_{3}$ are shown in the supplementary material. In each $\mathrm{CdS} / \mathrm{VO}_{\mathrm{x}}$ bilayer, two $\mathrm{Au}(40 \mathrm{~nm})$ electrodes were patterned on top of the CdS/ $\mathrm{VO}_{\mathrm{x}}$ heterostructures films. XRD measurements were done in a Rigaku SmartLab system at room temperature. Single-phase growth is confirmed by $\mathrm{XRD}$, epitaxially along the $\langle 012\rangle$ direction for $\mathrm{V}_{2} \mathrm{O}_{3}$, textured along $\langle 002\rangle$ for $\mathrm{VO}_{2}$, and hexagonal phase direction $\mathrm{H}\langle 002\rangle$ for CdS. In all samples, negligible changes in the crystal structure were observed upon exposing them to light (see supplementary material). Transport measurements were carried out on a Montana C2 S50 Cryocooler and TTPX Lakeshore cryogenic probe station, using a Keithley 6221 current source and a Keithley 2182A nanovoltmeter. A white LED (Thorlabs model MCWHLP1) was used for the photoconductivity measurements.

Figure 2 shows the hysteretic resistance of the samples as a function of temperature in the dark, due to the first order MIT of over five orders of magnitude in $\mathrm{CdS} / \mathrm{V}_{2} \mathrm{O}_{3}$ and three orders of magnitude in $\mathrm{CdS} / \mathrm{VO}_{2}$. This temperature-dependent behavior is identical to that of bare $\mathrm{VO}_{2}$ and $\mathrm{V}_{2} \mathrm{O}_{3}$, shown in Fig. 3, confirming that growing CdS over the vanadium oxide does not modify their properties. Figure 2 shows the effect of illumination of these samples with varying light power densities. As the light power density is increased, the MIT temperature is reduced and the resistance of the insulating ground state decreases. The effects are most notable in the $\mathrm{CdS} / \mathrm{V}_{2} \mathrm{O}_{3}$ bilayer, where the MIT is almost completely suppressed for a light power density of $731 \mathrm{~mW} / \mathrm{cm}^{2}$. This results in a resistance drop of more than 6 orders of magnitude compared to the case with no light. The light effects are smaller, though still significant, for $\mathrm{VO}_{2}$ with $\mathrm{T}_{\mathrm{MIT}}$ decreasing by $\sim 15 \mathrm{~K}$ when illuminated with a power of $731 \mathrm{~mW} / \mathrm{cm}^{2}$, meaning a drop of about two orders of magnitude in the resistance at $308 \mathrm{~K}$.


FIG. 2. Light-induced modification of the metal-insulator transition in $\mathrm{CdS} /$ vanadium oxide heterostructures. Electrical transport measurements of the resistance as a function of temperature from the bilayers (a) $\mathrm{CdS}_{2} \mathrm{O}_{3}$ and (b) $\mathrm{CdS} / \mathrm{NO}_{2}$. (c) Values of metal-insulating transition temperatures ( $\mathrm{T}_{\mathrm{MIT}}$ ) plotted as a function of the power density of the light source. 
a)

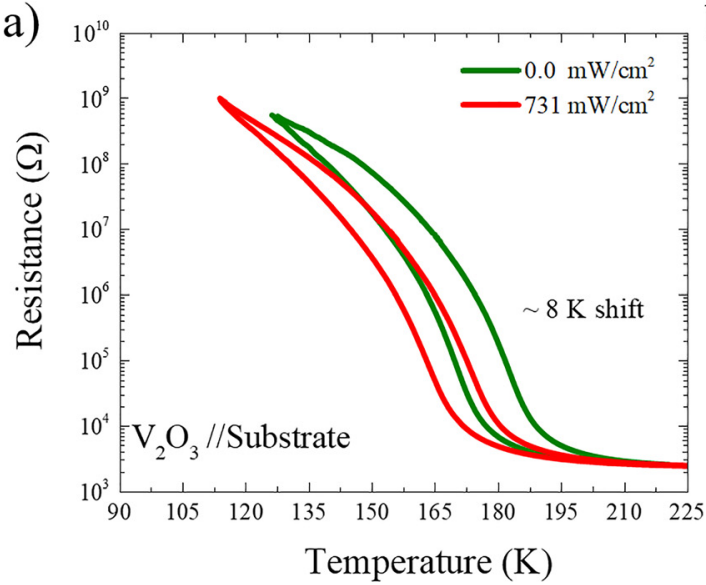

b)

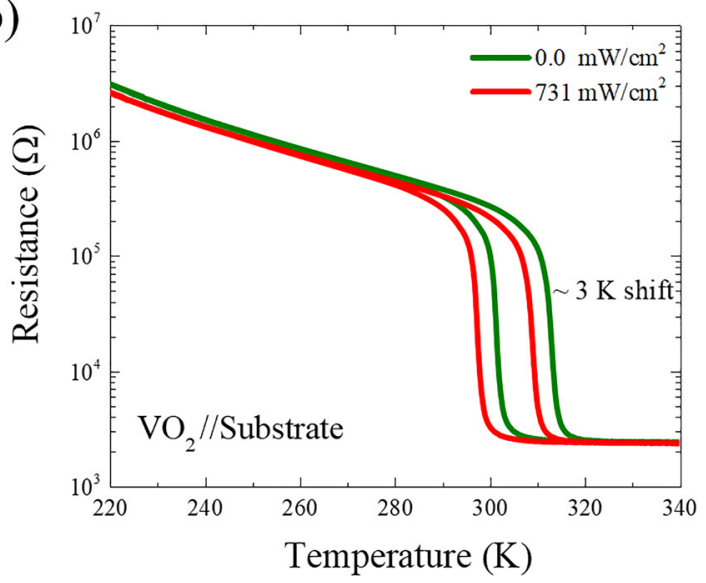

FIG. 3. Light-induced modulation of the metal-insulator transition in bare vanadium oxide films. Electrical transport measurements of the resistance as a function of the temperature in bare vanadium oxide thin film without $\mathrm{CdS}$ : (a) $\mathrm{V}_{2} \mathrm{O}_{3}(10 \pm 1 \mathrm{~nm})$ and (b) $\mathrm{VO}_{2}(10 \pm 1 \mathrm{~nm})$. The green curve corresponds to resistance vs temperature without light and the red curve with light. In both cases, a small shift in the onset of the transition is labeled.

In the absence of CdS, the effect of light directly on the vanadium oxide is much smaller, as shown in Fig. 3. This implies that the CdS plays a crucial role in our observations and that sample heating does not play a role. To investigate further the origin of this effect, we repeated the photodoping experiments with thicker $\mathrm{VO}_{\mathrm{X}}$ films $(100 \pm 2 \mathrm{~nm})$, which exhibit no changes in the $\mathrm{T}_{\mathrm{MIT}}$ (see the supplementary material). This points to a proximity effect localized at the $\mathrm{CdS} / \mathrm{VO}_{\mathrm{X}}$ interface. To further corroborate this, we fabricated samples in which a $10-30 \mathrm{~nm}$ thick, insulating $\mathrm{Al}_{2} \mathrm{O}_{3}$ layer was sandwiched between the vanadium oxide and the CdS (see the supplementary material), so that any proximity effect would be suppressed. No MIT modification was observed in this case.

There are additional major differences between the response of the two CdS/vanadium oxide hybrid heterostructures. Figure 4 shows the resistance vs temperature ( $\mathrm{R}$ vs $\mathrm{T}$ ) for the two types of heterostructures, measured with and without light, as a function of time. The green curves show the $\mathrm{R}$ vs $\mathrm{T}$ before illuminating the samples, the red curves during illumination, and the black dashed curves show the state after turning the light off. The $\mathrm{V}_{2} \mathrm{O}_{3}$ bilayer presents a volatile modulation of the MIT [Fig. 4(a)], i.e., the R vs T recovers its original shape immediately after the light is switched off. This contrasts to the nonvolatile modulation in the $\mathrm{VO}_{2}$. The $\mathrm{R}$ vs $\mathrm{T}$ does not recover its original shape, and the MIT remains suppressed for hours after the light has been turned off. This nonvolatile change, however, is not permanent. The blue curve shows that after $8 \mathrm{~h}$ at room temperature, the original MIT returns to its initial behavior. More information can be found in the supplementary material. Importantly, this demonstrates that the photodoping process does not introduce permanent damage into the sample.

A qualitative explanation of these effects arises by considering the charge carriers in the CdS and $\mathrm{VO}_{\mathrm{X}}$ as well as the fact that Mott MITs are highly sensitive to the carrier concentration. ${ }^{36}$ It is well
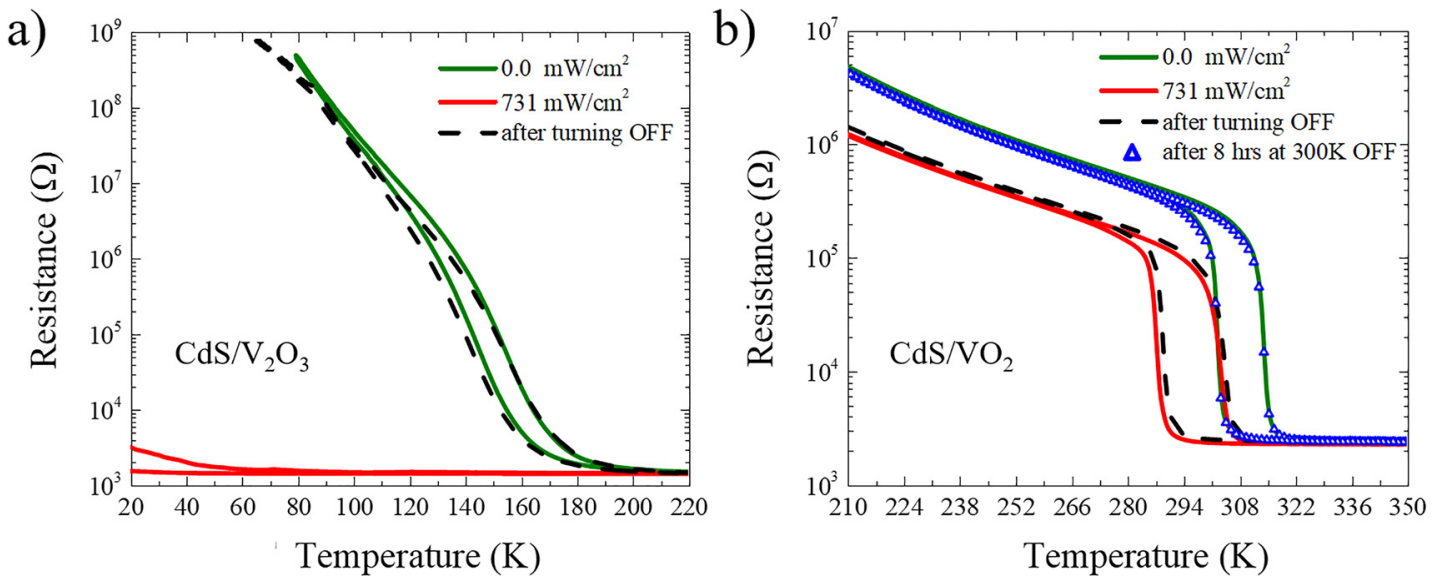

FIG. 4. Time dependence of the light-induced modulation. (a) Volatile changes in the transition temperature of the $\mathrm{CdS} / \mathrm{V}_{2} \mathrm{O}_{3}$ heterostructure. (b) Nonvolatile changes observed in the $\mathrm{CdS} / \mathrm{NO}_{2}$ sample. In both cases, the dash lines show the resistance vs temperature immediately after turning off the light. In VO the recovery of the original state after $8 \mathrm{~h}$ at room temperature. 
documented that conductivity of CdS in the photo-excited state is through electron carriers. ${ }^{37}$ On the other hand, while in $\mathrm{V}_{2} \mathrm{O}_{3}$, the electrical conduction is by holes ${ }^{38}$ and that of the $\mathrm{VO}_{2}$ is by electrons. ${ }^{39}$ The critical carrier concentration needed to undergo a Mott MIT can be affected by adding more photoelectrically created electrons from the $\mathrm{CdS}$, which leak into the $\mathrm{VO}_{\mathrm{X}}$. If the work function of the CdS falls between that of $\mathrm{V}_{2} \mathrm{O}_{3}$ and $\mathrm{VO}_{2}$, the observed differences in the effect would have a natural explanation. This would explain qualitatively three important facts discovered in our experiments: (1) the effect is an interface effect, (2) the light completely disrupts the MIT in $\mathrm{V}_{2} \mathrm{O}_{3}$ and has a minor effect on the $\mathrm{VO}_{2}$, and (3) the effect is volatile in $\mathrm{V}_{2} \mathrm{O}_{3}$ and nonvolatile in $\mathrm{VO}_{2}$.

Alternatively, the differences observed may suggest that the underlying mechanism for the MIT modulation in the various CdS/VOx samples is different. The nonvolatile modulation observed in $\mathrm{VO}_{2}$ could be explained by light-induced chemical reactions. On the other hand, CdS is also one of the most prominent photocatalysts. ${ }^{34,40}$ Irradiation with photons of energy larger than the bandgap may create electron-hole pairs, which would produce reduction/oxidation reactions at the $\mathrm{CdS} / \mathrm{VO}_{\mathrm{X}}$ interface. This would reduce the $\mathrm{VO}_{2}$ into a different Magnéli phase. ${ }^{41}$ After the light is switched off, those chemical changes would persist and only slowly reverse over time. The volatile $\mathrm{V}_{2} \mathrm{O}_{3}$ case is different and would be compatible with a modulation of the MIT caused by photodoping. Electrons generated by light in the CdS would transfer across the interface into the ultrathin (hole conducting) $\mathrm{V}_{2} \mathrm{O}_{3}$ layer, doping it and destabilizing the Mott insulating phase. Future work will be performed to identify which of these speculative scenarios lead to the effect we observe.

In conclusion, we have demonstrated large, light-induced modulation of the MIT in $\mathrm{CdS} / \mathrm{VO}_{\mathrm{X}}$ heterostructures. For CdS/ $\mathrm{V}_{2} \mathrm{O}_{3}$, the $\mathrm{T}_{\text {MIT }}$ modulation is as large as $140 \mathrm{~K}$, and the giant resistance reduction is as high as six orders of magnitude. For CdS/ $\mathrm{VO}_{2}$, the modulation is much smaller, although the resistance can be reduced by two orders of magnitude in the middle of the transition. A single study showed that light irradiation can be used to modify the insulating resistivity of $\mathrm{VO}_{2},{ }^{30}$ with minimal effect on the transition temperature. The modification we observe is volatile in $\mathrm{V}_{2} \mathrm{O}_{3}$ bilayers and nonvolatile in $\mathrm{VO}_{2}$ bilayers. We show that these effects are caused by the intimate contact at the CdS/vanadium oxide interface. A simple qualitative explanation suggests that these effects are caused by the differences in the electronic carriers in CdS (electrons), $\mathrm{V}_{2} \mathrm{O}_{3}$ (holes), and $\mathrm{VO}_{2}$ (electrons) and their work functions. An alternative possible explanation for these effects involves photodoping in $\mathrm{V}_{2} \mathrm{O}_{3}$, ${ }^{42}$ and CdS-mediated photocatalysis in $\mathrm{VO}_{2} \cdot{ }^{43,44}$ Further experimental work is under way to elucidate the precise mechanism. The volatile and nonvolatile behavior in two closely related material systems opens up the possibility for the use of these systems for different neuromorphic applications such as synaptors and neuristors.

In a broader sense, our results show a very promising approach toward the development of hybrid functionalities in materials using the possible transfer of electronic responses in a properly engineered heterostructure. This may have further applications as functional materials useful in other optoelectronic applications or systems where a different functionality can affect each other when incorporated into hybrid heterostructures.
See the supplementary material related to the structural characterization of the analyzed heterostructures.

We thank R. C. Dynes, A. Hoffmann, J. A. Schuller, and Y. Takamura for useful conversations. We thank Francisco Schuller for supplying the Au for the electrodes. This collaborative work was supported as part of the "Quantum Materials for Energy Efficient Neuromorphic Computing" (Q-MEEN-C), an Energy Frontier Research Center funded by the U.S. Department of Energy, Office of Science, Basic Energy Sciences under the Award No. DESC0019273. A.R.-C. thanks the economic support of the mobility research program Salvador de Madariaga from Spanish Ministry of Science.

I.K.S. and H.N. conceived the idea. H.N. and J.d.V. designed the experiment. H.N. and Y.K. fabricated the samples. H.N. performed the transport measurements with assistance from J.d.V., N.M.V., A.R.C., P.L., and E.Q. The x-rays diffraction measurements were performed by H.N., C.A., M.H.L., and I.Z.; I.Z., O.S., and A.F. carried out the x-ray diffraction analysis. H.N., A.F., and I.K.S. wrote the manuscript. All authors participated in the discussion of the results and corrected multiple iterations of the manuscript.

\section{DATA AVAILABILITY}

The data that support the findings of this study are available from the corresponding author upon reasonable request.

\section{REFERENCES}

${ }^{1}$ D. G. Schlom, L.-Q. Chen, C.-B. Eom, K. M. Rabe, S. K. Streiffer, and J.-M. Triscone, Annu. Rev. Mater. Res. 37(1), 589-626 (2007).

${ }^{2}$ M. Imada, A. Fujimori, and Y. Tokura, Rev. Mod. Phys. 70(4), 1039-1263 (1998).

${ }^{3}$ J. H. Ngai, F. J. Walker, and C. H. Ahn, Annu. Rev. Mater. Res. 44(1), 1-17 (2014).

${ }^{4}$ A. Sawa, Mater. Today 11(6), 28-36 (2008).

${ }^{5}$ N. F. Quackenbush, J. W. Tashman, J. A. Mundy, S. Sallis, H. Paik, R. Misra, J. A. Moyer, J. H. Guo, D. A. Fischer, J. C. Woicik, D. A. Muller, D. G. Schlom, and L. F. J. Piper, Nano Lett. 13(10), 4857-4861 (2013).

${ }^{6}$ Z. Yang, C. Ko, and S. Ramanathan, Annu. Rev. Mater. Res. 41(1), 337-367 (2011).

${ }^{7}$ J. Wei, Z. Wang, W. Chen, and D. H. Cobden, Nat. Nanotechnol. 4(7), 420-424 (2009).

${ }^{8}$ A. Beck, J. G. Bednorz, C. Gerber, C. Rossel, and D. Widmer, Appl. Phys. Lett. 77(1), 139-141 (2000).

${ }^{9}$ K. Liu, S. Lee, S. Yang, O. Delaire, and J. Wu, Mater. Today 21(8), 875-896 (2018).

${ }^{10}$ C. G. Granqvist and G. A. Niklasson, Buildings 7(1), 3 (2016).

${ }^{11}$ A. Zylbersztejn and N. F. Mott, Phys. Rev. B 11(11), 4383-4395 (1975).

${ }^{12}$ S. Kumar, M. D. Pickett, J. P. Strachan, G. Gibson, Y. Nishi, and R. S. Williams, Adv. Mater. 25(42), 6128-6132 (2013).

${ }^{13}$ S. Chen, Z. Wang, L. Fan, Y. Chen, H. Ren, H. Ji, D. Natelson, Y. Huang, J. Jiang, and C. Zou, Phys. Rev. B 96(12), 125130 (2017).

${ }^{14}$ M. Yang, Y. Yang, H. Bin, L. Wang, K. Hu, Y. Dong, H. Xu, H. Huang, J. Zhao, H. Chen, L. Song, H. Ju, J. Zhu, J. Bao, X. Li, Y. Gu, T. Yang, X. Gao, Z. Luo, and C. Gao, Sci. Rep. 6(1), 23119 (2016).

${ }^{15}$ D. G. Schlom, L.-Q. Chen, C. J. Fennie, V. Gopalan, D. A. Muller, X. Pan, R. Ramesh, and R. Uecker, MRS Bull. 39(2), 118-130 (2014).

${ }^{16}$ N. B. Aetukuri, A. X. Gray, M. Drouard, M. Cossale, L. Gao, A. H. Reid, R. Kukreja, H. Ohldag, C. A. Jenkins, E. Arenholz, K. P. Roche, H. A. Dürr, M. G. Samant, and S. S. P. Parkin, Nat. Phys. 9(10), 661-666 (2013).

${ }^{17}$ Y. H. Matsuda, D. Nakamura, A. Ikeda, S. Takeyama, Y. Suga, H. Nakahara, and Y. Muraoka, Nat. Commun, 11(1), 3591 (2020).

${ }^{18}$ B. Wu, A. Zimmers, H. Aubin, R. Ghosh, Y. Liu, and R. Lopez, Phys. Rev. B 84(24), 241410 (2011). 
${ }^{19}$ J. Jeong, N. Aetukuri, T. Graf, T. D. Schladt, M. G. Samant, and S. S. P. Parkin, Science 339(6126), 1402-1405 (2013).

${ }^{20}$ C. Wan, E. H. Horak, J. King, J. Salman, Z. Zhang, Y. Zhou, P. Roney, B. Gundlach, S. Ramanathan, R. H. Goldsmith, and M. A. Kats, ACS Photonics 5(7), 2688-2692 (2018).

${ }^{21}$ K. Dong, S. Hong, Y. Deng, H. Ma, J. Li, X. Wang, J. Yeo, L. Wang, S. Lou, K. B. Tom, K. Liu, Z. You, Y. Wei, C. P. Grigoropoulos, J. Yao, and J. Wu, Adv. Mater. 30(5), 1703878 (2018).

${ }^{22}$ K. A. Hallman, K. J. Miller, A. Baydin, S. M. Weiss, and R. F. Haglund, Adv Opt. Mater. 9, 2001721 (2021).

${ }^{23}$ Y. Ke, S. Wang, G. Liu, M. Li, T. J. White, and Y. Long, Small 14(39), 1802025 (2018).

${ }^{24}$ A. Singer, J. G. Ramirez, I. Valmianski, D. Cela, N. Hua, R. Kukreja, J. Wingert, O. Kovalchuk, J. M. Glownia, M. Sikorski, M. Chollet, M. Holt, I. K. Schuller, and O. G. Shpyrko, Phys. Rev. Lett. 120(20), 207601 (2018).

${ }^{25}$ G. Xu, P. Jin, M. Tazawa, and K. Yoshimura, Appl. Surf. Sci. 244(1-4), 449-452 (2005).

${ }^{26}$ L. Fan, Y. Chen, Q. Liu, S. Chen, L. Zhu, Q. Meng, B. Wang, Q. Zhang, H. Ren, and C. Zou, ACS Appl. Mater. Interfaces 8(48), 32971-32977 (2016).

${ }^{27}$ P. Markov, R. E. Marvel, H. J. Conley, K. J. Miller, R. F. Haglund, and S. M. Weiss, ACS Photonics 2(8), 1175-1182 (2015).

${ }^{28}$ N. A. Butakov, M. W. Knight, T. Lewi, P. P. Iyer, D. Higgs, H. T. Chorsi, J. Trastoy, J. Del Valle Granda, I. Valmianski, C. Urban, Y. Kalcheim, P. Y. Wang, P. W. C. Hon, I. K. Schuller, and J. A. Schuller, ACS Photonics 5(10), 4056-4060 (2018)

${ }^{29}$ D. Lee, J. Lee, K. Song, F. Xue, S.-Y. Choi, Y. Ma, J. Podkaminer, D. Liu, S.-C. Liu, B. Chung, W. Fan, S. J. Cho, W. Zhou, J. Lee, L.-Q. Chen, S. H. Oh, Z. Ma, and C.-B. Eom, Nano Lett. 17(9), 5614-5619 (2017).

${ }^{30}$ Y. Muraoka, T. Yamauchi, Y. Ueda, and Z. Hiroi, J. Phys.: Condens. Matter 14(49), L757-L763 (2002).
${ }^{31}$ K. Deng and L. Li, Adv. Mater. 26(17), 2619-2635 (2014).

${ }^{32}$ S. Kose, F. Atay, V. Bilgin, I. Akyuz, and E. Ketenci, Appl. Surf. Sci. 256(13), 4299-4303 (2010).

${ }^{33} \mathrm{X}$. Cheng, S. C. Tjong, and R. K. Y. Li, "1-Synthesis and optical properties of cadmium sulfide/polymer nanocomposite particles," in Physical Properties and Applications of Polymer Nanocomposites, edited by S. C. Tjong et al. (Woodhead Publishing, 2010), pp. 3-30.

${ }^{34}$ L. Cheng, Q. Xiang, Y. Liao, and H. Zhang, Energy Environ. Sci. 11(6), 1362-1391 (2018).

${ }^{35}$ D. M. Meysing, M. M. Griffith, W. L. Rance, M. O. Reese, J. M. Burst, C. A. Wolden, and T. M. Barnes, "Properties of oxygenated cadmium sulfide (CdS:O) and their impact on CdTe device performance," in IEEE 40th Photovoltaic Specialist Conference (PVSC), 8-13 June 2014 (2014), pp. 0964-0967.

${ }^{36}$ F. Xia, Z. Shao, Y. He, R. Wang, X. Wu, T. Jiang, S. Duhm, J. Zhao, S.-T. Lee, and J. Jie, ACS Nano 10(11), 10283-10293 (2016).

${ }^{37}$ H. Fujita, K. Kobayashi, T. Kawai, and K. Shiga, J. Phys. Soc. Jpn. 20(1), 109-122 (1965).

${ }^{38}$ D. B. McWhan and J. P. Remeika, Phys. Rev. B 2(9), 3734-3750 (1970).

${ }^{39}$ D. Ruzmetov, D. Heiman, B. B. Claflin, V. Narayanamurti, and $S$. Ramanathan, Phys. Rev. B 79(15), 153107 (2009).

${ }^{40} \mathrm{~N}$. Serpone and A. V. Emeline, J. Phys. Chem. Lett. 3(5), 673-677 (2012).

${ }^{41}$ U. Schwingenschlögl and V. Eyert, Ann. Phys. 13(9), 475-510 (2004).

${ }^{42}$ M. K. Liu, B. Pardo, J. Zhang, M. M. Qazilbash, S. J. Yun, Z. Fei, J.-H. Shin, H.-T. Kim, D. N. Basov, and R. D. Averitt, Phys. Rev. Lett. 107(6), 066403 (2011).

${ }^{43}$ Y. Wang, Z. Zhang, Y. Zhu, Z. Li, R. Vajtai, L. Ci, and P. M. Ajayan, ACS Nano 2(7), 1492-1496 (2008).

${ }^{44}$ L. Fan, Y. Zhu, S. Zhao, Z. Wang, Z. Liu, L. Zhu, B. Wang, and Q. Zhang, Sol. Energy Mater. Sol. Cells 212, 110562 (2020). 